\title{
SELF-SIMILARITY AND RANDOM WALKS
}

\author{
VADIM A. KAIMANOVICH
}

\begin{abstract}
This is an introductory level survey of some topics from a new branch of fractal analysis - the theory of self-similar groups. We discuss recent works on random walks on self-similar groups and their applications to the problem of amenability for these groups.
\end{abstract}

\section{INTRODUCTION}

The purpose of this paper is to give a brief survey of some ideas and methods associated with new progress in understanding the so-called self-similar groups. This class of groups consists of automorphisms of homogeneous rooted trees defined in a simple recursive way. A good account of the initial period of the theory can be found in the survey [BGN03] by Bartholdi, Grigorchuk and Nekrashevych and in the recent monograph of Nekrashevych [Nek05] (their authors are among the most active contributors to this field).

Self-similar groups have a natural interpretation in terms of fractal geometry; their limit sets are very interesting fractal sets (see the recent papers [NT08, RT09] for a study of Laplacians on limit sets). These groups also arise as iterated monodromy groups of rational endomorphisms of the Riemann sphere, which, for instance, led to a recent solution of an old problem from rational dynamics BN06.

Self-similar groups are often quite unusual from the point of view of the traditional group theory. This has both advantages and disadvantages. On one hand, by using selfsimilar groups it is easy to construct examples which may otherwise be much less accessible (for instance, the famous Grigorchuk group of intermediate growth [Gri80, Gri85, dlH00] has a very simple self-similar presentation). On the other hand, even the simplest group theoretical questions for self-similar groups may be quite hard. Already finding a selfsimilar realization of a free group is very far from being obvious [BS98, GM05, VV07.

Another question of this kind is whether a given self-similar group is amenable (amenability introduced by von Neumann [vN29] is, in a sense, the most natural generalization of finiteness, and it plays a fundamental role in group theory). There are numerous characterizations of amenability in various terms. In particular, it is known to be equivalent to existence of a random walk on the group with trivial behaviour at infinity (三 trivial Poisson boundary) [Fur73, Ros81, KV83. It turns out that self-similar groups may have random walks which are also self-similar in a certain sense, and it is this self-similarity that can be used in order to prove triviality of the Poisson boundary, and therefore establish amenability of the underlying group.

This idea was first used by Bartholdi and Viràg [BV05] for proving amenability of the socalled Basilica group $\mathcal{B}$. This group first studied by Grigorchuk and Żuk [GŻo2a] has a very simple matrix presentation and also arises as the iterated monodromy group of the map $z \mapsto z^{2}-1$ (known as the Basilica map, whence the name). In particular, Grigorchuk and $\dot{Z}$ uk proved that $\mathcal{B}$ is not subexponentially elementary, which made especially interesting the question about its amenability. The approach of Bartholdi and Viràg was further developed by Kaimanovich Kai05 who used the entropy theory of random walks (which provides a simple criterion of triviality of the Poisson boundary) in combination with a 
contraction property for the asymptotic entropy of random walks on self-similar groups (the "Münchhausen trick").

An important ingredient of this technique is a link (established in [Kai05) between self-similarity and the so-called random walks with internal degrees of freedom (RWIDF) [KS83] also known under the names of matrix-valued random walks [CW89] or of covering Markov chains Kai95. These are group invariant Markov chains which take place on the product of a group by a certain parameter set. Any random walk on a self-similar group naturally gives rise to a random walk with internal degrees of freedom parameterized by the alphabet of the action tree of the group. In turn, this RWIDF, when restricted to the copy of the group corresponding to a fixed value of the freedom parameter, produces a new random walk on the self-similar group (as it is pointed out in [GN07, this transformation corresponds to the classical operation of taking the Schur complement of a matrix). It is the interplay between the original and the new random walks, which allows one to apply the Münchhausen trick.

This technique was recently applied by Bartholdi, Kaimanovich and Nekrashevych [BKN08 to prove amenability of all self-similar groups generated by bounded automata (this class, in particular, contains the Basilica group).

In this survey we attempt to give a historical and conceptual overview of these developments without going into technical details, so that it should hopefully be suitable for a first acquaintance with the subject. Structurally, our presentation is split into three parts. In Section 1 we discuss the notion of self-similarity, introduce self-similar groups in general and the subclass of self-similar groups generated by bounded automata. Further in Section 2 we briefly discuss the notion of amenability of a group. Finally, in Section 3 we analyze random walks on self-similar groups, and show how they can be used for establishing amenability of self-similar groups.

The presentation is based on a talk given at the "Fractal Geometry and Stochastics IV" conference (and on several other occasions as well). I would like to thank the organizers of this meeting for a very interesting and inspiring week in Greifswald.

\section{SELF-SIMILAR GROUPS}

1.A. And so ad infinitum ... The modern idea of self-similarity is best described by the following quote from On Poetry: a Rhapsody by Jonathan Swift (1733)]:

So, naturalists observe, a flea

Has smaller fleas that on him prey;

And these have smaller still to bite 'em,

And so proceed ad infinitum.

On a more formal level, the simplest self-similarity assumptions are:

- a part is similar to the whole;

- the whole is a union of such parts;

- these parts are pairwise disjoint.

\footnotetext{
${ }^{1}$ It was naturally extended by Augustus de Morgan in his Budget of Paradoxes (1872):

Great fleas have little fleas upon their backs to bite 'em,

And little fleas have lesser fleas, and so ad infinitum.

And the great fleas themselves, in turn have, greater fleas to go on;

While these again have greater still, and greater still, and so on.
}

This is a description of what is nowadays called the natural extension of a non-invertible dynamical system. See Kai03 for its applications in the context of fractal sets. 
These assumptions naturally lead to the most fundamental self-similar structure, that of a rooted homogeneous tree. Such trees are, for instance, skeletons of iterated function systems satisfying the open set condition (e.g., see [Fal03]), the simplest one of which is the classical Cantor set. More precisely, let $X \cong\{1,2, \ldots, d\}$ be a finite set called the alphabet. Denote by $X^{*}$ the set of all finite words in the alphabet $X$ (including the empty word $\varnothing$ ). In other terms, $X^{*}$ is the free monoid generated by the set $X$ (the composition being the concatenation $\left.\left(w, w^{\prime}\right) \mapsto w w^{\prime}\right)$. The associated rooted homogeneous tree $T=T(X)$ is the (right) Cayley graph of the free monoid $X^{*}$ (so that one connects $w$ to $w x$ by an edge for all $w \in X^{*}, x \in X$ ). The tree $T(X) \cong X^{*}$ is split into levels $T_{n} \cong X^{n}$ (the set of words of length $n$ ). The level $T_{0}$ consists only of the empty word $\varnothing$, which is the root of $T$. Each vertex $w \in T \cong X^{*}$ is the root of the subtree $T_{w}$ which consists of all the words beginning with $w$. The map $w^{\prime} \mapsto w w^{\prime}$ provides then a canonical identification of the trees $T$ and $T_{w}$, see Figure 1, where $X=\{a, b\}$.

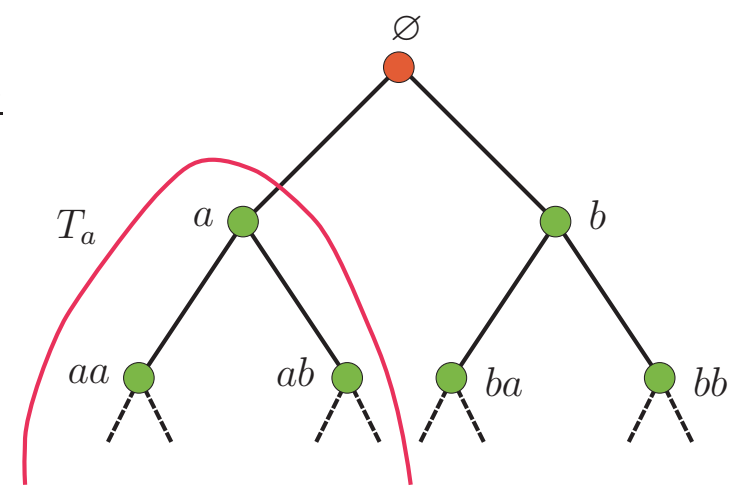

Figure 1.

1.B. Generalized permutation matrices. The group $\mathfrak{G}=\operatorname{Aut}(T)$ of automorphisms of the tree $T$ obviously preserves each level of $T$. In particular, it acts by permutations on the first level $X \cong T_{1}$, i.e., there is a homomorphism $g \mapsto \sigma=\sigma^{g}$ from $\mathfrak{G}$ to $\operatorname{Sym}(X)$ (the permutation group on $X$ ). Another piece of data associated with any automorphism $g \in \mathfrak{G}$ is a collection of automorphisms $\left\{g_{x}\right\}_{x \in X}$ indexed by the alphabet $X$. Indeed, if $y=\sigma^{g}(x)$, then $g$ establishes a one-to-one correspondence between the corresponding subtrees $T_{x}$ and $T_{y}$ rooted at the points $x$ and $y$, respectively. Since both subtrees $T_{x}$ and $T_{y}$ are canonically isomorphic to the full tree $T$, the map $g: T_{x} \rightarrow T_{y}$ is conjugate to an automorphism of $T$ denoted by $g_{x}$ (in terms of Swift's description above, $g_{x}$ describes what happens on the back of a first order flea when it moves from position $x$ to position $\left.y=\sigma^{g}(g)\right)$, see Figure 2 .

Conversely, a permutation $\sigma \in \operatorname{Sym}(X)$ and a collection $\left\{g_{x}\right\}_{x \in X}$ of elements of $\mathfrak{G}$ uniquely determine the associated automorphism $g \in \mathfrak{G}$. In algebraic terms it means that $g \mapsto\left(\sigma^{g} ;\left\{g_{x}\right\}_{x \in X}\right)$ is an isomorphism of the group $\mathfrak{G}$ and the semi-direct product $\operatorname{Sym}(X) \wedge \mathfrak{G}^{X}$ (in yet another terminology, $\mathfrak{G}$ is isomorphic to the permutational wreath product $\mathfrak{G} \geq \operatorname{Sym}(X))$. There is a very convenient way of visualizing this structure by means of generalized permutation matrices.

Recall that the usual permutation matrix $M^{\sigma}$ associated with a permutation $\sigma \in$ $\operatorname{Sym}(X)$ is a $|X| \times|X|$ matrix with entries

$$
M_{x y}^{\sigma}=\left\{\begin{array}{ll}
1, & \text { if } y=\sigma(x), \\
0, & \text { otherwise }
\end{array},\right.
$$




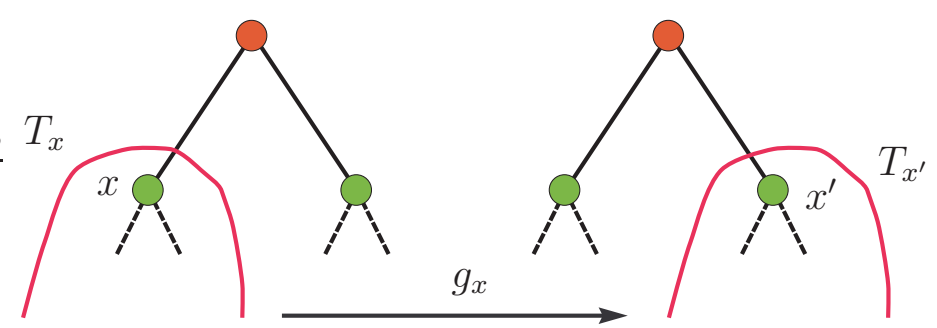

FiguRe 2.

and that the map $\sigma \mapsto M^{\sigma}$ is a group isomorphism. In the same way we shall present the data $\left(\sigma^{g} ;\left\{g_{x}\right\}_{x \in X}\right)$ by the generalized permutation matrix $M^{g}$ with entries

$$
M_{x y}^{g}= \begin{cases}g_{x}, & \text { if } y=\sigma^{g}(x) \\ 0, & \text { otherwise. }\end{cases}
$$

For instance, the automorphism $g$ described in Figure 3 is presented by the matrix

$$
M^{g}=\left(\begin{array}{cc}
0 & g_{1} \\
g_{2} & 0
\end{array}\right) .
$$

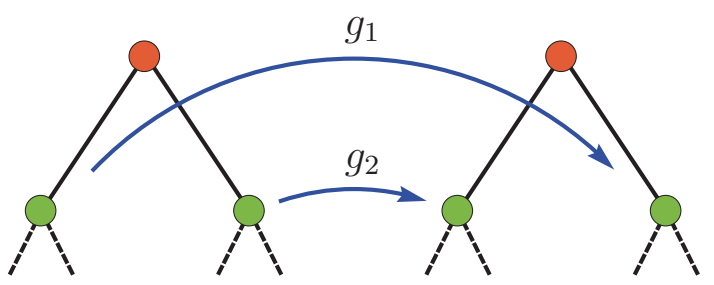

FiguRE 3.

More generally, given an arbitrary group $G$, we shall denote by

$$
\operatorname{Sym}(X ; G)=G \text { ২ } \operatorname{Sym}(X)=\operatorname{Sym}(X) \wedge G^{X}
$$

the group of generalized permutation matrices of order $|X|$ with non-zero entries from the group $G$. The group operation here is the usual matrix multiplication, the only difference with ordinary permutation matrices being that the matrix elements are multiplied according to the group law of $G$. Obviously, application of the augmentation map (which consists in replacing all group elements with 1) to a generalized permutation matrix yields a usual permutation matrix, which corresponds to the natural homomorphism of $\operatorname{Sym}(X ; G)$ onto $\operatorname{Sym}(X)$. We can now sum up the above discussion by saying that there is a natural isomorphism of the group $\mathfrak{G}=\operatorname{Aut}(T)$ of automorphisms of the tree $T=T(X)$ and of the generalized permutation group $\operatorname{Sym}(X ; \mathfrak{G})$. It is this isomorphism that embodies the self-similarity properties of the group $\mathfrak{G}$.

\section{C. Self-similar groups and matrix presentations.}

Definition. A countable subgroup $G \subset \mathfrak{G}$ is self-similar if the restriction of the isomorphism $\mathfrak{G} \rightarrow \operatorname{Sym}(X ; \mathfrak{G})$ to $G$ induces an embedding $G \hookrightarrow \operatorname{Sym}(X ; G)$; in other words, if all entries of the matrices $M^{g}, g \in G$ belong to $G$. Note that, rigorously speaking, self-similarity is a property of the embedding $G \subset \mathfrak{G}$ rather than of the group $G$ only. The embedding $G \hookrightarrow \operatorname{Sym}(X ; G)$ need not be surjective (see the example below). 
Example. The adding machine (isomorphic to the group $\mathbb{Z}$ ) is generated by the transformation $a: z \mapsto z+1$ on the ring $\mathbb{Z}_{2}$ of 2-adic integers $\varepsilon_{0}+\varepsilon_{1} \cdot 2+\cdots+\varepsilon_{n} \cdot 2^{n}+\ldots$, where the digits $\varepsilon_{i}$ take values 0 or 1 . Depending on the values of initial digits, it acts in the following way:

$$
\begin{aligned}
& 0 \varepsilon_{1} \varepsilon_{2} \varepsilon_{3} \ldots \mapsto \quad 1 \varepsilon_{1} \varepsilon_{2} \varepsilon_{3} \ldots, \\
& 10 \varepsilon_{2} \varepsilon_{3} \ldots \mapsto 01 \varepsilon_{2} \varepsilon_{3} \ldots \text {, } \\
& 110 \varepsilon_{3} \ldots \mapsto 001 \varepsilon_{3} \ldots \text {, } \\
& \text {... }
\end{aligned}
$$

We can think of sequences $\left(\varepsilon_{0}, \varepsilon_{1}, \ldots\right)$ as of boundary points of the binary rooted tree $T=$ $T(X)$ of the alphabet $X=\{0,1\}$. The transformation $a$ extends to an automorphism of $T$, and, as one can easily see from its symbolic description above, the associated generalized permutation matrix is

$$
M^{a}=\left(\begin{array}{ll}
0 & 1 \\
a & 0
\end{array}\right)
$$

Thus, the infinite cyclic group $\langle a\rangle \cong \mathbb{Z}$ generated by the transformation $a$ is self-similar (as a subgroup of the full group of automorphisms $\mathfrak{G}$ ).

Note that the automorphism $a$ is completely determined by the matrix $M^{a}$. Indeed, the augmentation map applied to $M^{a}$ produces the usual permutation matrix $\left(\begin{array}{ll}0 & 1 \\ 1 & 0\end{array}\right)$ which describes the permutation by which $a$ acts on the first level of the tree $T$. Further, by substituting $M^{a}$ for $a$ and the identity matrix for 1 in $M^{a}$ one obtains the matrix

$$
\left(\begin{array}{llll}
0 & 0 & 1 & 0 \\
0 & 0 & 0 & 1 \\
0 & 1 & 0 & 0 \\
a & 0 & 0 & 0
\end{array}\right)
$$

augmentation of which produces the order 4 permutation matrix

$$
\left(\begin{array}{llll}
0 & 0 & 1 & 0 \\
0 & 0 & 0 & 1 \\
0 & 1 & 0 & 0 \\
1 & 0 & 0 & 0
\end{array}\right)
$$

describing the action of $a$ on the second level of $T$. By recursively repeating this procedure one obtains the action of $a$ on all levels of $T$, i.e., an automorphism of the whole tree $T$.

The example above suggests the following way of defining self-similar groups by their matrix presentations. Fix a finite (or countable, for infinitely generated groups) set $K$ (the future set of generators of a self-similar group), and assign to each $\kappa \in K$ a generalized permutation matrix $M^{\kappa}$ whose non-zero entries are words in the alphabet consisting of letters from $K$ and their inverses (the entry associated with the empty word is 1). By replacing the non-zero entries of matrices $M^{\kappa}$ with corresponding products of the associated matrices and their inverses we obtain generalized permutation matrices of order $|X|^{2}$, etc. The usual permutation matrices obtained from them by the augmentation map determine then the action of elements from $K$ on all levels of the tree $T$, i.e., the corresponding automorphisms of $T$. See [BG00] or [Nek05] for more on recursions of this kind.

A particular case of this construction arises in the situation when all non-zero entries of matrices $M^{\kappa}$ are elements of the set $K$. In this case the assignment $\kappa \mapsto M^{\kappa}$ amounts to a map $(\kappa, x) \mapsto(\lambda, y)$ of the product $K \times X$ to itself, i.e., to an automaton. Here $y=\sigma(x)$ 
for the permutation $\sigma=\sigma\left(M^{\kappa}\right)$ determined by the matrix $M^{\kappa}$, and $\lambda$ is the matrix entry $M_{x y}^{\kappa}$. The self-similar group obtained in this way is called an automaton group 2

1.D. The Basilica group. The Basilica group $\mathcal{B}$ is determined by the matrix presentation

$$
a \mapsto\left(\begin{array}{cc}
b & 0 \\
0 & 1
\end{array}\right), \quad b \mapsto\left(\begin{array}{cc}
0 & a \\
1 & 0
\end{array}\right)
$$

The aforementioned recursion for this group looks in the following way:

$$
\begin{aligned}
& a \mapsto\left(\begin{array}{cc}
b & 0 \\
0 & 1
\end{array}\right) \mapsto\left(\begin{array}{cccc}
0 & a & 0 & 0 \\
1 & 0 & 0 & 0 \\
0 & 0 & 1 & 0 \\
0 & 0 & 0 & 1
\end{array}\right) \mapsto \ldots, \\
& b \mapsto\left(\begin{array}{ll}
0 & a \\
1 & 0
\end{array}\right) \mapsto\left(\begin{array}{llll}
0 & 0 & b & 0 \\
0 & 0 & 0 & 1 \\
1 & 0 & 0 & 0 \\
0 & 1 & 0 & 0
\end{array}\right) \mapsto \ldots,
\end{aligned}
$$

and the associated automaton is

$$
\left\{\begin{aligned}
(a, 1) & \mapsto(b, 1) \\
(a, 2) & \mapsto(e, 2) \\
(b, 1) & \mapsto(a, 2) \\
(b, 2) & \mapsto(e, 1) \\
(e, 1) & \mapsto(e, 1) \\
(e, 2) & \mapsto(e, 2) .
\end{aligned}\right.
$$

Here $\{1,2\}=X$ is the alphabet of the binary tree $T$, and $K=\{a, b, e\}$, where $e$ is the group identity determined by the substitution $e \mapsto\left(\begin{array}{ll}e & 0 \\ 0 & e\end{array}\right)$.

The group $\mathcal{B}$ was first studied by Grigorchuk and Żuk GŻ02a] (see below for its algebraic propereties). The name Basilica comes from the fact that it also appears as the iterated monodromy group of the rational map $z \mapsto z^{2}-1$ on the Riemann sphere $\overline{\mathbb{C}}$.

This latter notion was introduced by Nekrashevych who created a very fruitful link between the theory of self-similar groups and rational dynamics. Namely, given a rational $\operatorname{map} \varphi: \overline{\mathbb{C}} \rightarrow \overline{\mathbb{C}}$ of degree $d$, a generic point $z \in \overline{\mathbb{C}}$ has precisely $d$ preimages, each of which also has $d$ preimages, etc. Thus, attached to a generic point $z \in \overline{\mathbb{C}}$ is the rooted homogeneous tree $T_{z}$ of its preimages. One can move the preimage tree along any continuous curve consisting of generic points. However, if $z$ follows a non-contractible loop, it may happen that, although the preimage tree $T_{z}$ returns to its original position, it undergoes a certain non-trivial monodromy transformation. Thus, there is a homomorphism of the fundamental group of the connected component of $z$ in the set of generic points to the group of automorphisms of the preimage tree $T_{z}$. The resulting subgroup of Aut $\left(T_{z}\right)$ is called the iterated monodromy group of the map $\varphi$, see [Nek05 for more details.

Now, in rational dynamics the map $z \mapsto z^{2}-1$ is called the Basilica map [Bie90, because its Julia set (a subset of the Riemann sphere which, in a sense, consists of the limit points of this map) looks similar to Basilica di San Marco in Venice (together with

\footnotetext{
${ }^{2}$ Usually, when talking about automata groups, one tacitly assumes that the corresponding automaton is finite, i.e., the generating set $K$ is finite.
} 
its reflection in the water), see Figure 4. This Julia set also arises as the limit set of the Basilica group $\mathcal{B}$.

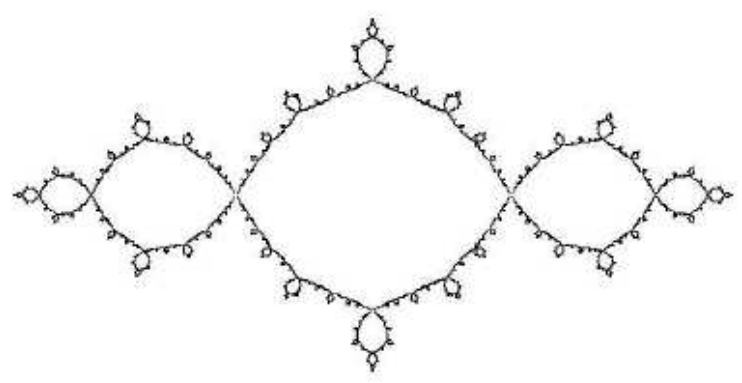

FiguRE 4.

Interesting algebraic and analytic properties of the Basilica group obtained in Ġ̈02a, GŻ02b, BG02 made especially relevant the question about its amenability formulated in GŻ02a, also see BGN03.

1.E. Bounded automatic automorphisms and Mother groups. The Basilica group $\mathcal{B}$ actually belongs to a certain natural subclass of the class of self-similar groups which we shall now describe.

As we have seen in Section 1.B, given an automorphism $g \in \mathfrak{G}=\operatorname{Aut}(T)$, any symbol $x \in X$ determines an associated automorphism $g_{x} \in \mathfrak{G}$. In the same way such an automorphism $g_{w} \in \mathfrak{G}$ (the state of $g$ at the point $w$ ) can be defined for an arbitrary word $w \in T \cong X^{*}$, by restricting the automorphism $g$ to the subtree $T_{w}$ with the subsequent identification of both $T_{w}$ and its image $g\left(T_{w}\right)=T_{g(w)}$ with $T$. Equivalently, $g_{w}$ can be obtained by recursively applying the presentation $g \mapsto M^{g}$, namely, $g_{w}$ is the nonzero entry of the row $R_{w}$ of the $|X|^{|w|} \times|X|^{|w|}$ matrix obtained by recursive expansion $g \mapsto M^{g} \mapsto \ldots$

If the set of states of $g$

$$
\mathrm{S}(g)=\left\{g_{w}: w \in T\right\} \subset \mathfrak{G}
$$

is finite, then the automorphism $g$ is called automatic. The set of all automatic automorphisms of the tree $T$ forms a countable subgroup $\mathfrak{A}=\mathfrak{A}(X)$ of $\mathfrak{G}=\mathfrak{G}(X)$.

An automorphism $g$ is called bounded if the sets $\left\{w \in X^{n}: g_{w} \neq e\right\}$ have uniformly bounded cardinalities over all $n$. The set of all bounded automorphisms also forms a subgroup $\mathfrak{B}=\mathfrak{B}(X)$ of $\mathfrak{G}=\mathfrak{G}(X)$. We denote by $\mathfrak{B A}=\mathfrak{B A}(X)=\mathfrak{B}(X) \cap \mathfrak{A}(X)$ the group of all bounded automatic automorphisms of the homogeneous rooted tree $T$.

It is easy to see that the generators $a, b$ of the Basilica group $\mathcal{B}$ described in Section 1.D are both automatic and bounded in the above sense, so that $\mathcal{B} \subset \mathfrak{B A}$ (here and on several occasions below we omit the alphabet $X$ from our notation). More generally, any group generated by a (finite) automaton (see Section [1.C) is a subgroup of $\mathfrak{A}$. An automaton is called bounded if its group is contained in $\mathfrak{B}$ (therefore, in $\mathfrak{B A}$ ). The class of groups generated by bounded automata was defined by Sidki in [Sid00]. Obviously, all these groups are subgroups of $\mathfrak{B A}$. Most of the well-studied groups of finite automata belong to this class (see [BKN08] for examples).

Groups generated by bounded automata also appear naturally in connection with fractal geometry. It was proved in BN03] that every such group is contracting, and a contracting group is generated by bounded automata if and only if the boundary of its tile is finite. 
This technical condition implies that the limit space (see [Nek05]) of such a group belongs to the well studied class of nested fractals (see [Kig01]). This is the class of fractals on which the Brownian motion is best understood. This shows an interesting connection between the most well-studied class of self-similar groups and the class of fractals with most well-understood analysis (see [NT08] for more details).

It turns out that the class of groups generated by bounded automata contains a countable family of groups which have certain universality properties with respect to this class.

Let $X$ be a finite set with a distinguished element $o \in X$, and put $\bar{X}=X \backslash\{o\}$. Set $A=\operatorname{Sym}(X)$ and $B=\operatorname{Sym}(\bar{X} ; A)$, and recursively embed the groups $A$ and $B$ into $\mathfrak{G}(X)$ by the matrix presentations

$$
M^{a}=\phi_{A}(a), \quad M^{b}=\left(\begin{array}{cc}
b & 0 \\
0 & \phi_{B}(b)
\end{array}\right),
$$

where $\phi_{A}(a), \phi_{B}(b)$ are, respectively, the permutation and the generalized permutation matrices corresponding to $a \in A, b \in B$. Then the Mother group $\mathfrak{M}=\mathfrak{M}(X)=\langle A, B\rangle$ is the subgroup of $\mathfrak{G}=\mathfrak{G}(X)$ generated by the finite groups $A$ and $B$.

A direct verification shows that both groups $A, B$ are contained in $\mathfrak{B A}$, whence the group $\mathfrak{M}(X)$ is a subgroup of $\mathfrak{B A}(X)$. On the other hand, as it was proved in [BKN08], any finitely generated subgroup of $\mathfrak{B A}(X)$ can be embedded as a subgroup into the generalized permutation group $\operatorname{Sym}\left(X^{N} ; \mathfrak{M}\left(X^{N}\right)\right)$ for some integer $N$.

Thus, in view of the fact that amenability is preserved by elementary operations (see Section 2.C below), amenability of the groups $\mathfrak{B A}(X)$ for all finite sets $X$ (therefore, amenability of all groups generated by bounded automata) would follow from amenability just of all the Mother groups $\mathfrak{M}(X)$.

It is worth noting that the groups generated by bounded automata form a subclass of the class of contracting self-similar groups (see [BN03, Nek05]). It is still an open question whether all contracting groups are amenable. However, Nekrashevych Nek08. recently established a weaker property: contracting groups contain no free groups with $\geq 2$ generators.

\section{Amenability}

2.A. From finite to infinite: von Neumann, Day and Reiter. Finite groups can be characterized as those discrete groups which have a finite invariant measure. In other words, a discrete group $G$ is finite if and only if the natural action of $G$ by translations on the space $\ell_{+, 1}^{1}(G)$ of positive normalized elements from $\ell^{1}(G)$ has a fixed point. This trivial observation suggests two ways of "extending" the finiteness property to infinite groups. One can look either for fixed points in a bigger space, or for approximative invariance instead of exact one.

The first idea was implemented by John von Neumann [vN29], according to whose definition amenable groups are those which admit a translation invariant mean 3 . A mean on $G$ is a finitely additive probability measure, in other words, an element of the space $\left[\ell^{\infty}\right]_{+, 1}^{*}$ of positive normalized functionals on $\ell^{\infty}(G)$. Usual measures on $G$ are also means, but if $G$ is infinite, then there are many more means than measures (which corresponds to the fact that in the infinite case $\ell^{1}$ is significantly "smaller" than its second dual space $\left.\left[\ell^{1}\right]^{* *}=\left[\ell^{\infty}\right]^{*}\right)$.

\footnotetext{
${ }^{3}$ Actually, the original term used by von Neumann was the German meßbare Gruppe, which means "measurable group" in English. It was later replaced in German with mittelbare (cf. moyennable in
} 
Means being highly non-constructive objects, the other way was explored (surprisingly, much later than the original definition of von Neumann) by Reiter [Rei65] who introduced (under the name $P_{1}$ ) what is nowadays known as Reiter's condition for a group $G$ : there exists an approximatively invariant sequence of probability measures on $G$, in other words, there exists a sequence of probability measures $\lambda_{n}$ on $G$ such that

$$
\left\|\lambda_{n}-g \lambda_{n}\right\| \underset{n \rightarrow \infty}{\longrightarrow} 0 \quad \forall g \in G
$$

where $\|\cdot\|$ denotes the total variation norm. He proved that the above condition is in fact equivalent to amenability as defined by von Neumann.

Example. The sequence of Cesaro averaging measures

$$
\lambda_{n}=\frac{1}{n+1}\left(\delta_{0}+\delta_{1}+\cdots+\delta_{n}\right)
$$

on the group of integers $\mathbb{Z}$ is approximatively invariant (here $\delta_{n}$ denotes the unit mass at the point $n$ ). Thus, $\mathbb{Z}$ is amenable.

2.B. Other definitions. There is a lot of other (equivalent) definitions of amenability of a countable group, which illustrates importance and naturalness of this notion. We shall briefly mention just some of them, referring the reader to [Gre69], Pie84 and Pat88] for more details. Moreover, the notion of amenability has been extended to objects other than groups, in particular, to group actions, equivalence relations, and, more generally, to groupoids (e.g., see [ADR00]).

The main application of the notion of amenability is its characterization as a fixed point property. Namely, a countable group $G$ is amenable if and only if any continuous affine action of $G$ on a compact space has a fixed point. An example of such an action arises in the following way. Let $X$ be a compact topological space endowed with a continuous action of $G$, and let $\mathcal{P}(X)$ denote the space of probability measures on $X$ endowed with the weak* topology. Then $\mathcal{P}(X)$ has a natural affine structure, and the action of $G$ extends to a continuous affine action on $\mathcal{P}(X)$. Therefore, any continuous action of an amenable group on a compact space has a finite invariant measure4 (in fact, this property can also be shown to be equivalent to amenability). In the case of the group of integers $\mathbb{Z}$ this result is known as the Krylov-Bogolyubov theorem [KB37] (which is one of the starting points of the modern theory of topological dynamical systems).

Yet another characterization of amenable groups can be given in terms of their isoperimetric properties. This condition is basically a specialization of Reiter's condition to sequences of measures of special kind (although historically it was introduced by Følner Føl55 some 10 years before Reiter). Let $A_{n}$ be a sequence of finite subsets of $G$, and let $\lambda_{n}$ be the associated uniform probability measures on $A_{n}$. Then Reiter's condition for

French), literally meaning "averageable". In English, however, Mahlon M. Day suggested to use (apparently, first as a pun) the word amenable, which appeared in print in this context for the first time in 1949 Day49. It is curious that Day himself, when he later described the history of this term in Day83 on the occasion of the nomination of his paper Day57 as a "Citation Classic" dated its appearance to 1955: In 1929, von Neumann studied a new class of groups, those with invariant means on the bounded functions. My thesis (1939) studied semigroups with invariant means; thereafter, I worked in the field alternately with the geometry of Banach spaces. I finished a large geometrical project in 1955 and turned back to invariant means; in order to talk to my students I invented the term 'amenable (pronounced as amean'able) semigroups'.

${ }^{4}$ The first proof of this fact by Bogolyubov Bog39 published in 1939 (immediately after [KB37]) in a rather obscure journal in Ukrainian remained almost unknown, see Ano94, CSGdlH99. 
the sequence $\lambda_{n}$ is equivalent to the following condition on the sets $A_{n}$ :

$$
\frac{\left|g A_{n} \triangle A_{n}\right|}{\left|A_{n}\right|} \underset{n \rightarrow \infty}{\longrightarrow} 0 \quad \forall g \in G,
$$

where $\triangle$ denotes the symmetric difference of two sets, and $|A|$ is the cardinality of a finite set $A$. A sequence of sets $A_{n}$ satisfying the above condition is called a Følner sequence, and the condition itself is called Følner's condition. Obviously, Følner's condition implies Reiter's condition; however, the usual "slicing" isoperimetric techniques also allow one to prove the converse, so that Følner's condition is equivalent to amenability.

For finitely generated groups Følner's condition takes especially simple form. Indeed, in this case it is enough to verify it for the elements $g$ from a finite generating set $K \subset G$ only. Let us assume that $K$ is symmetric, and denote by $\Gamma=\Gamma(G, K)$ the (left) Cayley graph of the group $G$ determined by $K$ (i.e., the vertex set is $G$, and the edges are of the form $(g, k g)$ with $g \in G$ and $k \in K)$. For a set $A \subset G$ denote by $\partial A=\partial_{K} A \subset A$ its boundary in the Cayley graph, i.e., the set of all points from $A$ which have a neighbor from the complement of $A$. Then a sequence of sets $A_{n} \subset G$ is $\mathrm{F} \varnothing l$ lner if and only if

$$
\frac{\left|\partial A_{n}\right|}{\left|A_{n}\right|} \underset{n \rightarrow \infty}{\longrightarrow} 0
$$

Existence of a sequence of sets as above is an isoperimetric characterization of amenability.

Example. For the group $\mathbb{Z}$ with the standard generating set $\{ \pm 1\}$ the boundary of the segment $A_{n}=\{0,1,2, \ldots, n\}$ consists of two points $\{0, n\}$, whereas $\left|A_{n}\right| \rightarrow \infty$, so that $\left\{A_{n}\right\}$ is a Følner sequence.

2.C. Elementary groups. The class of amenable groups is closed with respect to the "elementary" operations of taking subgroups, quotients, extensions and inductive limits. Finite and abelian groups are amenable (cf. Example). The minimal class of groups containing finite and abelian groups and closed with respect to the above elementary operations is called elementary amenable (EA) Day57.

In the above paper Day asked the question whether every amenable group is elementary amenable. The first example of an amenable but not elementary amenable group is the group of intermediate growth (see below) found by Grigorchuk Gri80, Gri85. Later, a finitely presented amenable extension of the Grigorchuk group was constructed in Gri98.

However, there is yet another way to obtain "obviously amenable" groups. It is related with the notion of growth. Let $G$ be a finitely generated group with a symmetric generating set $K$. Denote by $B_{n}$ the $n$-ball of the Cayley graph metric on $G$ centered at the group identity, or, in other words, the set of all elements of $G$ which can be presented as products of not more than $n$ generators from $K$. The sequence $\left|B_{n}\right|$ is submultiplicative, so that there exists a $\operatorname{limit} \lim \log \left|B_{n}\right| / n$. The group $G$ is said to have exponential or subexponential growth depending on whether this limit is positive or zero (this property does not depend on the choice of a generating set $K$ ).

The class of groups of subexponential growth contains all the groups of polynomial growth (the ones for which $\left|B_{n}\right|$ is bounded from above by a polynomial function; by a theorem of Gromov [Gro81] these are precisely finite extensions of nilpotent groups), but there are also examples of groups of intermediate growth, i.e., the ones whose growth is subexponential without being polynomial. First examples of this kind were constructed by Grigorchuk [Gri85], and these groups can often be realized as self-similar groups (see

\footnotetext{
${ }^{5}$ Elsewhere in this paper we shall always deal with the right Cayley graphs. However, in order to keep the notations consistent, here it is more convenient to consider the left Cayley graphs.
} 
[BGN03]). For instance, the most famous of the Grigorchuk groups has 4 generators acting on the rooted binary tree with the matrix presentation

$$
a \mapsto\left(\begin{array}{ll}
0 & 1 \\
1 & 0
\end{array}\right), \quad b \mapsto\left(\begin{array}{cc}
a & 0 \\
0 & c
\end{array}\right), \quad c \mapsto\left(\begin{array}{ll}
a & 0 \\
0 & d
\end{array}\right), \quad d \mapsto\left(\begin{array}{ll}
1 & 0 \\
0 & b
\end{array}\right) .
$$

If the growth of $G$ is subexponential, then the sequence $B_{n}$ necessarily contains a Følner subsequence, so that the groups of subexponential growth are amenable. Therefore, one can change the definition of elementary amenable groups by extending the set of "building blocks": the minimal class of groups containing finite, abelian and subexponential groups and closed with respect to the elementary operations is called subexponentially elementary amenable (SEA). Thus, a natural goal is to find amenable groups which are not subexponentially elementary (see Gri98, CSGdlH99]).

It is the Basilica group $\mathcal{B}$ which provided the first example of this kind. It was shown in Ġं02a] that it does not belong to the class SEA, whereas it was proved in BV05] that the Basilica group is amenable. We shall now explain the role of random walks in the proof of amenability of $\mathcal{B}$.

Remark. It is worth mentioning at this point that non-amenable groups do exist, and there is actually quite a lot of them (for instance, numerous matrix groups: non-elementary Fuchsian and Kleinian groups, lattices in semi-simple Lie groups, etc.). The first example is of course the free group $\mathcal{F}_{d}$ with $d \geq 2$ generators (since any discrete group can be obtained from free groups by the elementary operations above, amenability of free groups would have implied amenability of all groups). Non-amenability of $\mathcal{F}_{d}$ may be explained in many different ways by using various definitions. For instance, it is not hard to build a paradoxical decomposition of $\mathcal{F}_{d}$ (see CSGdlH99] and the references therein), which prevents it from having an invariant mean. Another way consists in noticing that there are continuous actions of $\mathcal{F}_{d}$ on compact sets admitting no finite invariant measures. Let, for instance $d=2$. Then an action of $\mathcal{F}_{2}$ on a compact $K$ is determined by specifying two homeomorphisms of $K$ corresponding to the generators of $\mathcal{F}_{2}$. If $K$ is the circle, then the only measure preserved by any irrational rotation is the Lebesgue measure. Take such a rotation for the first homeomorphism from the definition of the action. Then, if we choose the second homeomorphism in such a way that it does not preserve the Lebesgue measure, then these two homeomorphisms (therefore, the associated action of $\mathcal{F}_{2}$ ) do not have any invariant measure, so that $\mathcal{F}_{2}$ is not amenable.

\section{RANDOM WALKS}

3.A. Convolution powers. The main idea behind the use of random walks for establishing amenability at first glance looks counterproductive. Let us replace arbitrary approximatively invariant sequences of probability measures $\lambda_{n}$ on $G$ from Reiter's condition with sequences of very special form, namely, with sequences of convolution powers $\mu^{* n}$ of a single probability measure $\mu$ on $G$. In the same way as with Følner's characterization of amenability (see Section 2.B), it turns out that this restricted class of approximatively invariant sequences is still sufficient in order to characterize amenability. More precisely, a group $G$ is amenable if and only if there exists a probability measure $\mu$ on $G$ such that the sequence of its convolution powers $\mu^{* n}$ is approximatively invariant. In one direction (the one we need for proving amenability) this is just a particular case of Reiter's condition, whereas in the opposite direction it was conjectured by Furstenberg [Fur73] and later independently proved by Kaimanovich-Vershik [VK79, KV83] and by Rosenblatt Ros81]. 
Now, working with sequences of convolution powers instead of arbitrary sequences of probability measures on $G$ is actually easier, because of their description as onedimensional distributions of random walks on $G$. Moreover, one can use a very powerful quantitative criterion of whether a given sequence of convolution powers is approximatively invariant. It is provided by the entropy theory of random walks which we shall briefly describe below.

Random walks are in a sense the most homogeneous Markov chains: they are homogeneous both in space and in time. The latter property means that the assignment $x \mapsto \pi_{x}$ of transition probabilities to the points from the state space is equivariant with respect to a group action, and the simplest instance of such an action is, of course, the action of a group on itself.

More formally, the (right) random walk on a countable group $G$ determined by a probability measure $\mu$ is the Markov chain with the state space $G$ and the transition probabilities

$$
\pi_{g}\left(g^{\prime}\right)=\mu\left(g^{-1} g^{\prime}\right)
$$

equivariant with respect to the left action of the group on itself. In other words, from a point $g$ the random walk moves at the next moment of time to the point $g h$, where the random increment $h$ is chosen according to the distribution $\mu$. We shall use for this description of transition probabilities of the random walk $(G, \mu)$ the notation

$$
g \stackrel{\longmapsto \sim \mu}{\longmapsto} g h .
$$

Thus, if the random walk starts at moment 0 from a point $g_{0}$, then its position at time $n$ is

$$
g_{n}=g_{0} h_{1} h_{2} \ldots h_{n}
$$

where $h_{i}$ is a Bernoulli sequence of independent $\mu$-distributed increments. Therefore, the distribution of the position $g_{n}$ of this random walk at time $n$ is the translate $g_{0} \mu^{* n}$ of the $n$-fold convolution power $\mu^{* n}$ of the measure $\mu$ (the convolution of two probability measures $\mu_{1}, \mu_{2}$ on $G$ is defined as the image of the product measure $\mu_{1} \otimes \mu_{2}$ on $G \times G$ under the map $\left.\left(g_{1}, g_{2}\right) \mapsto g_{1} g_{2}\right)$.

3.B. Trivial future. Reiter's condition

$$
\left\|g \mu^{* n}-\mu^{* n}\right\| \underset{n \rightarrow \infty}{\longrightarrow} 0 \quad \forall g \in G
$$

for the sequence of convolution powers $\mu^{* n}$ means that the time $n$ one-dimensional distributions of the random walk issued from the group identity $e$ and from an arbitrary point $g \in G$ asymptotically coincide. Probabilistically, the fact that the one-dimensional distributions of a Markov chain asymptotically do not depend on their starting points means that the "remote future" (behaviour at infinity) of the chain does not depend on its present, which, in view of the classical Kolmogorov argument means that the "remote future" must be trivial.

In order to explain the latter notion in a more rigorous way, let us look at two examples: the simple random walks on the 2-dimensional integer lattice (三 the Cayley graph of the group $\mathbb{Z}^{2}$ ) and on the homogeneous tree of degree 4 (三 the Cayley graph of the free group $\mathcal{F}_{2}$ with 2 generators). The simple random walk on a graph is the one whose transition probabilities are equidistributed among neighbours; the simple random walk on the Cayley graph of a group is precisely the random walk on this group determined by the probability measure $\mu=\mu_{K}$ equidistributed on the generating set $K$. Locally, each of these graphs is regular of degree 4 (each point has 4 neighbours), but their global geometry is very different, see Figure 5. In particular, the Cayley graph of $\mathcal{F}_{2}$ is endowed with a natural 
boundary $\partial \mathcal{F}_{2}$ which can, for instance, be identified with the space of all paths without backtracking issued from the identity of the group (or any other reference point).
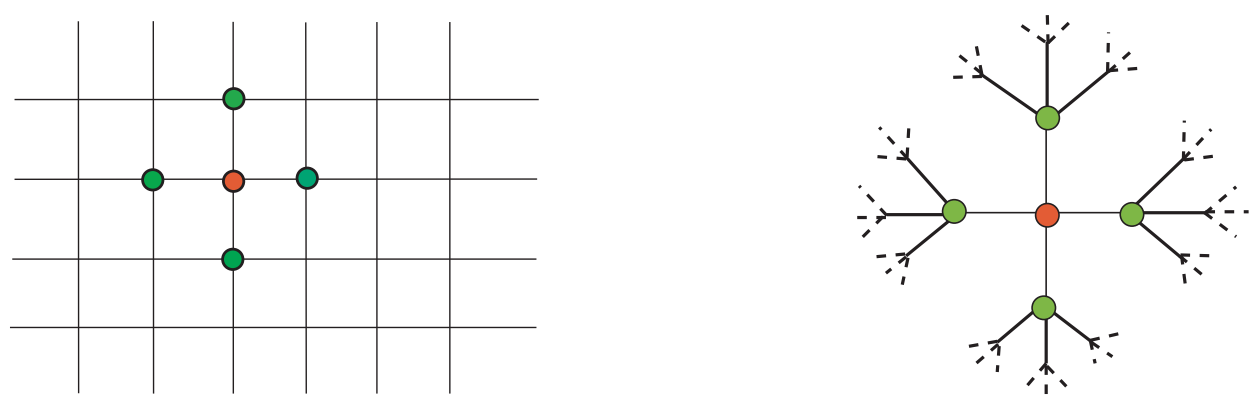

\section{FiguRE 5.}

The global behaviour of sample paths of simple random walks on these graphs is also very different. Sample paths of the simple random walk on $\mathcal{F}_{2}$ converge a.s. to the boundary $\partial \mathcal{F}_{2}$ (of course, different sample paths may converges to different limits). Thus, these limit points can be used to distinguish sample paths by their behaviour at infinity. On the other hand, although sample paths of the random walk on $\mathbb{Z}^{2}$ are quite complicated (their scaling limit is the Brownian motion on the plane), they all look "the same", see Figure 6.
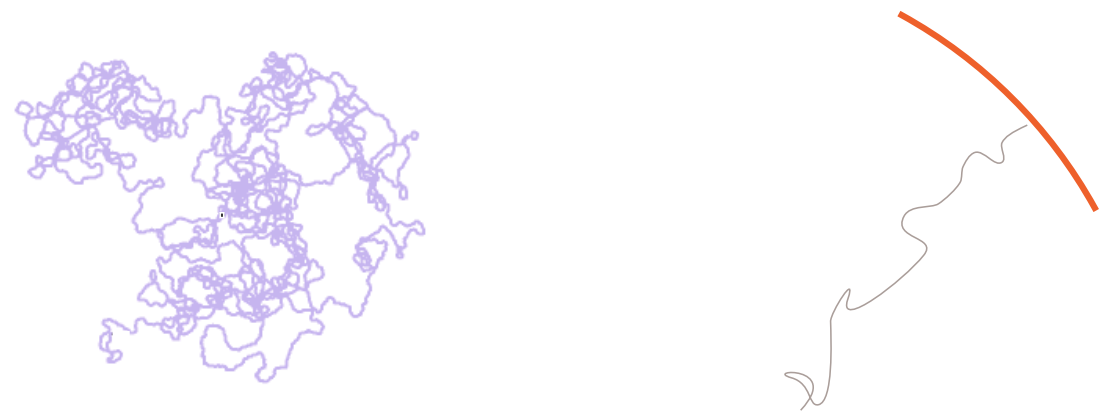

FiguRE 6.

Formally speaking, the "remote future" of a Markov chain is described by its tail $\sigma$ algebra

$$
\mathfrak{A}^{\infty}=\bigcap_{n} \mathfrak{A}_{n}^{\infty}
$$

which is the limit of the decreasing sequence of $\sigma$-algebras $\mathfrak{A}_{n}^{\infty}$ generated by the positions of the chain at times $\geq n$. Thus, the boundary convergence of sample paths of the simple random walk on $\mathcal{F}_{2}$ at once implies non-triviality of its tail $\sigma$-algebra. On the other hand, in spite of absence of any visible behavior at infinity, proving triviality of the tail $\sigma$-algebra for the simple random walk on $\mathbb{Z}^{2}$ requires additional work.

On a formal level a criterion of the triviality of the tail $\sigma$-algebra of an arbitrary Markov chain is provided by the corresponding 0-2 law Der76, Kai92. Its "zero part" for the random walk on a group $G$ determined by a probability measure $\mu$ takes the following form (see [KV83]): the tail $\sigma$-algebra of the random walk is trivial if and only if

$$
\left\|g \mu^{* n}-\mu^{*(n+1)}\right\| \underset{n \rightarrow \infty}{\longrightarrow} 0 \quad \forall g \in \operatorname{supp} \mu .
$$


Thus, if the support of the measure $\mu$ generates $G$ as a group, and the tail $\sigma$-algebra of the random walk $(G, \mu)$ is trivial, then the sequence of Cesaro averages of the convolution powers satisfies Reiter's condition, and therefore the group $G$ is amenable (actually, if $\mu$ is aperiodic, then the sequence of convolution powers $\mu^{* n}$ also satisfies Reiter's condition).

3.C. Asymptotic entropy. Let $p=\left(p_{i}\right)$ be a discrete probability distribution. By Shannon the amount of information contained in any outcome $i$ is $-\log p_{i}$. The average amount of information per outcome

$$
H(p)=-\sum p_{i} \log p_{i}
$$

is called the entropy of the distribution $p$. The entropy has the following two properties (by which it is essentially characterized, see [YY83]):

- monotonocity: if $p^{\prime}$ is a quotient of the distribution $p$ (i.e., $p^{\prime}$ is obtained by gluing together some of the states of $p$ ), then

$$
H\left(p^{\prime}\right)<H(p),
$$

- additivity: for any two distributions $p_{1}, p_{2}$ the entropy of their product is

$$
H\left(p_{1} \otimes p_{2}\right)=H\left(p_{1}\right)+H\left(p_{2}\right) .
$$

Let $\mu$ be a probability measure on a countable group $G$ with finite entropy $H(\mu)$. The (asymptotic) entropy of the random walk $(G, \mu)$ is defined as

$$
h(G, \mu)=\lim _{n \rightarrow \infty} \frac{H\left(\mu^{* n}\right)}{n},
$$

in other words, $h(G, \mu)$ is the asymptotic mean specific amount of information about a single increment $h_{i}$ in the product $g_{n}=h_{1} h_{2} \ldots h_{n}$. Existence of the above limit follows from the fact that the sequence $H\left(\mu^{* n}\right)$ is subadditive. Indeed, by the definition of convolution, the measure $\mu^{*(n+m)}$ is a quotient of the product measure $\mu^{* n} \otimes \mu^{* m}$, whence

$$
H\left(\mu^{*(n+m)}\right) \leq H\left(\mu^{* n} \otimes \mu^{* m}\right)=H\left(\mu^{* n}\right)+H\left(\mu^{* m}\right)
$$

by the above monotonicity and additivity properties.

The asymptotic entropy was first introduced by Avez Ave72. As it turned out, it plays a crucial role in understanding the asymptotic properties of the random walk $(G, \mu)$. Namely, as it was independently proved by Kaimanovich-Vershik [VK79, KV83] and Derriennic [Der80, the asymptotic entropy $h(G, \mu)$ vanishes if and only if the tail $\sigma$ algebra of the random walk $(G, \mu)$ is trivial.

This result means that the "remote future" of the random walk $(G, \mu)$ is trivial if and only if the amount of information about the first increment $h_{1}$ in the random product $g_{n}=h_{1} h_{2} \ldots h_{n}$ asymptotically vanishes. Indeed, on a more rigorous level, this amount of information is the mean conditional entropy (see [Roh67]) $H\left(h_{1} \mid g_{n}\right)$ of $h_{1}$ with respect to $g_{n}$. It can be easily seen to coincide with the difference $H\left(\mu^{* n}\right)-H\left(\mu^{*(n-1)}\right)$ between the entropies of two consecutive convolution powers, whence the claim.

3.D. Self-similarity and RWIDF. A random walk with internal degrees of freedom (RWIDF) on a group $G$ is a Markov chain whose state space is the product of $G$ by a certain space $X$ (the space of degrees of freedom), and its transition probabilities are equivariant with respect to the action of $G$ on itself. Thus, the transition probabilities are

$$
(g, x) \stackrel{\mu_{x y}(h)}{\longmapsto}(g h, y)
$$


where $M=\left\{\mu_{x y}: x, y \in X\right\}$ is a $|X| \times|X|$ matrix of subprobability measures on $G$ such that

$$
\sum_{y}\left\|\mu_{x y}\right\|=1 \quad \forall x \in X .
$$

The image of the RWIDF $(G, M)$ under the map $(g, x) \mapsto x$ is the quotient Markov chain on $X$ with the transition matrix

$$
P=\left(p_{x y}\right), \quad p_{x y}=\left\|\mu_{x y}\right\|
$$

which is the image of the matrix $M$ under the augmentation map.

There is a natural link between random walks on self-similar groups and random walks with internal degrees of freedom. Let $\mu$ be a probability measure on a self-similar group $G$. Then the associated random walk is

$$
g \stackrel{\mu(h)}{\longmapsto} g h .
$$

By using the self-similar embedding $G \hookrightarrow \operatorname{Sym}(X ; G)$ it gives rise to the random walk on the generalized permutation group $\operatorname{Sym}(X ; G)$ with the transition probabilities

$$
M^{g} \stackrel{\mu(h)}{\longmapsto} M^{g} M^{h} \text {. }
$$

Since multiplication of the matrix $M^{g} \in \operatorname{Sym}(X ; G)$ by the increment $M^{h}$ is done row by row, we obtain the following Markov chain on the space of these rows:

$$
R \stackrel{\mu(h)}{\longmapsto} R M^{h}
$$

Due to the definition of the group $\operatorname{Sym}(X ; G)$ the rows of the corresponding matrices can be identified with points of the product space $G \times X$ (each row has precisely one non-zero entry, so that it is completely described by the value of this entry and by its position). Therefore, the latter Markov chain can be interpreted as a Markov chain on $G \times X$ whose transition probabilities are easily seen to be invariant with respect to the left action of $G$ on $G \times X$, i.e., as a random walk on $G$ with internal degrees of freedom parameterized by the alphabet $X$. This RWIDF is described by the transition probabilities matrix

$$
M^{\mu}=\left(\mu_{x y}\right)=\sum_{g} \mu(g) M^{g} .
$$

Recall that stopping a Markov chain at the times when it visits a certain recurrent subset of the state space produces a new Markov chain on this recurrent subset (the trace of the original Markov chain). For a random walk with internal degrees of freedom on $G \times X$ determined by a matrix $M$ take for such a subset the copy $G \times\{x\}$ of the group $G$ obtained by fixing a value $x \in X$. It is recurrent provided the quotient chain on $X$ is irreducible. The transition probabilities of the induced chain on $G \times\{x\}$ are obviously equivariant with respect to the left action of the group $G$ on itself (because the original RWIDF also has this property). Therefore, the induced chain on $G \times\{x\}$ is actually the usual random walk determined by a certain probability measure $\mu^{x}$ on $G$.

The measures $\mu^{x}, x \in X$ can be expressed in terms of the matrix $M$ as

$$
\begin{aligned}
\mu^{x} & =\mu_{x x}+M_{x \bar{x}}\left(I+M_{\overline{x x}}+M_{\overline{x x}}^{2}+\ldots\right) M_{\bar{x} x} \\
& =\mu_{x x}+M_{x \bar{x}}\left(I-M_{\overline{x x}}\right)^{-1} M_{\bar{x} x},
\end{aligned}
$$

where $M_{x \bar{x}}$ (resp., $M_{\bar{x} x}$ ) denotes the row $\left(\mu_{x y}\right)_{y \neq x}$ (resp., the column $\left(\mu_{y x}\right)_{y \neq x}$ ) of the matrix $M$ with the removed element $\mu_{x x}$, and $M_{\overline{x x}}$ is the $(d-1) \times(d-1)$ matrix (where 
$d=|X|)$ obtained from $M$ by removing its $x$-th row and column. The multiplication above is understood in the usual matrix sense 6

This is elementary probability. We look at the quotient chain on $X$ and replace its transition probabilities $p_{x y}$ with the transition measures $\mu_{x y}$ in the identity

$$
\begin{aligned}
1 & =p_{x x}+\sum_{n=0}^{\infty} \sum_{y_{0}, \ldots, y_{n} \neq x} p_{x y_{0}} p_{y_{0} y_{1}} \cdots p_{y_{n-1} y_{n}} p_{y_{n} x} \\
& =p_{x x}+P_{x \bar{x}}\left(I+P_{\overline{x x}}+P_{\overline{x x}}^{2}+\ldots\right) P_{\bar{x} x} \\
& =p_{x x}+P_{x \bar{x}}\left(I-P_{\overline{x x}}\right)^{-1} P_{\bar{x} x},
\end{aligned}
$$

which yields

$$
\begin{aligned}
\mu^{x} & =\mu_{x x}+\sum_{n=0}^{\infty} \sum_{y_{0}, \ldots, y_{n} \neq x} \mu_{x y_{0}} \mu_{y_{0} y_{1}} \cdots \mu_{y_{n-1} y_{n}} \mu_{y_{n} x} \\
& =\mu_{x x}+M_{x \bar{x}}\left(I+M_{\overline{x x}}+M_{\overline{x x}}^{2}+\ldots\right) M_{\bar{x} x} \\
& =\mu_{x x}+M_{x \bar{x}}\left(I-M_{\bar{x} x}\right)^{-1} M_{\bar{x} x} .
\end{aligned}
$$

The first term in this formula corresponds to staying at the point $x$ (and performing on $G$ the jump determined by the measure $\mu_{x x}$ ), whereas in the second term the first factor corresponds to moving from $x$ to $X \backslash\{x\}$, the second one to staying in $X \backslash\{x\}$ (each matrix power $M_{x x}^{n}$ corresponding to staying in $X \backslash\{x\}$ for precisely $n$ steps), and the third one to moving back from $X \backslash\{x\}$ to the point $x$. The matrix notation automatically takes care of what is going on with the $G$-component of the RWIDF.

The measures $\mu^{x}$ also admit the following interpretation in terms of the original random walk $(G, \mu)$ with the sample paths $\left(g_{n}\right)$ : we look at it only at the moments $n$ when $g_{n}\left(T_{x}\right)=$ $T_{x}$, and $\mu^{x}$ is then the law of the induced random walk on the group $\operatorname{Aut}\left(T_{x}\right) \cong \operatorname{Aut}(T)$.

3.E. The Münchhausen trick. Let us now look at what happens with the asymptotic entropy in the course of the transformations

$$
\mu \mapsto M=M^{\mu} \mapsto \mu^{x}
$$

Since the information contained in a matrix does not exceed the sum of informations about each row, passing to asymptotic entropies we obtain the inequality

$$
h(G, \mu) \leq d h(G, M) .
$$

Further, all points $x \in X$ are visited by RWIDF $(G, M)$ with the same asymptotic frequency $1 / d$ (because the uniform distribution is stationary for the quotient chain on $X$ ), whence

$$
h\left(G, \mu^{x}\right)=d h(G, M) \geq h(G, \mu) .
$$

The idea of the Münchhausen trick [Kai05] consists in combining two observations. The first one is the above entropy inequality. The second observation is that if the measure $\mu^{x}$ is a non-trivial convex combination

$$
\mu^{x}=(1-\alpha) \delta_{e}+\alpha \mu, \quad 0<\alpha<1
$$

of the original measure $\mu$ and the $\delta$-measure at the identity of the group (in which case we call the measure $\mu$ self-similar $)$, then

$$
h\left(G, \mu^{x}\right)=\alpha h(G, \mu) .
$$

\footnotetext{
${ }^{6}$ As it is pointed out in GN07, this formula corresponds to the classical operation of taking the Schur complement of a matrix.
} 
The result of these two observations is the inequality

$$
h(G, \mu) \leq \alpha h(G, \mu) .
$$

Taken into account that $0<\alpha<1$, it is only possible if $h(G, \mu)=0$, which proves amenability of the group $G$.

We shall now show how the Münchhausen trick works in two particular cases: for the Basilica group $\mathcal{B}$ and for the Mother groups $\mathfrak{M}$.

3.F. The Basilica group. As we have seen in Section 1.D, the Basilica group is defined by the matrix presentation

$$
\mathcal{B}: a \mapsto\left(\begin{array}{cc}
b & 0 \\
0 & 1
\end{array}\right), \quad b \mapsto\left(\begin{array}{ll}
0 & a \\
1 & 0
\end{array}\right)
$$

Take on $\mathcal{B}$ a symmetric probability measure $\mu$ supported by the generators $a, b$ and their inverses:

$$
\mu=\alpha\left(a+a^{-1}\right)+\beta\left(b+b^{-1}\right),
$$

for $\alpha, \beta>0$ such that $2(\alpha+\beta)=1$. The matrix associated with the measure $\mu$ is

$$
\begin{aligned}
M^{\mu} & =\alpha M^{a}+\alpha M^{a^{-1}}+\beta M^{b}+\beta M^{b^{-1}} \\
& =\alpha\left(\begin{array}{ll}
b & 0 \\
0 & 1
\end{array}\right)+\alpha\left(\begin{array}{cc}
b^{-1} & 0 \\
0 & 1
\end{array}\right)+\beta\left(\begin{array}{ll}
0 & a \\
1 & 0
\end{array}\right)+\beta\left(\begin{array}{cc}
0 & 1 \\
a^{-1} & 0
\end{array}\right) \\
& =\left(\begin{array}{cc}
\alpha\left(b+b^{-1}\right) & \beta(1+a) \\
\beta\left(1+a^{-1}\right) & 2 \alpha
\end{array}\right) .
\end{aligned}
$$

Therefore, the trace of the RWIDF $\left(\mathcal{B}, M^{\mu}\right)$ on the copy of $\mathcal{B}$ corresponding to the first letter 1 of the 2-letter alphabet $\{1,2\}$ is the random walk governed by the measure

$$
\begin{aligned}
\widetilde{\mu}=\widetilde{\mu}^{1} & =\mu_{11}+\mu_{12}\left(1-\mu_{22}\right)^{-1} \mu_{21} \\
& =\alpha\left(b+b^{-1}\right)+\frac{\beta}{2}(1+a)\left(1+a^{-1}\right)=\beta+\frac{\beta}{2}\left(a+a^{-1}\right)+\alpha\left(b+b^{-1}\right) .
\end{aligned}
$$

If

$$
\frac{\alpha}{\beta}=\frac{\beta}{2 \alpha} \Longleftrightarrow 2 \alpha^{2}=\beta^{2}
$$

then

$$
\widetilde{\mu}=\beta+(1-\beta) \mu
$$

so that the measure $\mu$ is self-similar, and Münchhausen's trick is applicable.

3.G. The Mother group. For proving amenability of the Mother group $\mathfrak{M}$ (here and below we omit the alpabet $X$ ) one can apply an approach somewhat different from the one which was used above for the Basilica group. It is based on the fact that the Mother group $\mathfrak{M}$ is generated by two finite subgroups $A$ and $B$. We take for the measure $\mu$ the convolution product of the uniform measures $m_{A}$ and $m_{B}$ on these subgroups:

$$
\mu=m_{A} * m_{B} .
$$

\footnotetext{
${ }^{7}$ It is named after venerable Baron Münchhausen who "... once rode on a cannon-ball and next told about it. Another time he reported he had to get himself and the good horse he sat on, out of a quagmire by pulling his own hair till he saved himself and his horse..."

${ }^{8}$ For simplicity we pass from now on to the group algebra notations putting $g$ for a $\delta$-measure $\delta_{g}, g \neq e$ and just 1 for the $\delta$-measure $\delta_{e}$ concentrated at the identity of the group.
} 
Then the matrix $M^{\mu}$ has a very special form

$$
M^{\mu}=M^{\mu_{A}} M^{\mu_{B}}=E_{d}\left(\begin{array}{cc}
\mu_{B} & 0 \\
0 & \mu_{A} E_{d-1}
\end{array}\right),
$$

where $d=|X|$, and $E_{d}$ denotes the order $d$ matrix with entries $1 / d$, so that $M^{\mu}$ has identical rows with entries

$$
M_{x y}^{\mu}= \begin{cases}\mu_{B} / d & \text { if } y=o, \\ \mu_{A} / d & \text { otherwise } .\end{cases}
$$

It means that transition probabilities of the associated RWIDF $\left(\mathfrak{M} \times X, M^{\mu}\right)$ do not depend on $x$, so that its projection to $\mathfrak{M}$ is just the random walk $(\mathfrak{M}, \widetilde{\mu})$ determined by the measure

$$
\widetilde{\mu}=\sum_{y} M_{x y}^{\mu}=\frac{d-1}{d} \mu_{A}+\frac{1}{d} \mu_{B},
$$

whereas the projection of RWIDF $\left(\mathfrak{M} \times X, M^{\mu}\right)$ to $X$ is the sequence of independent $X$-valued random variables with uniform distribution on $X$ (because all entries $M_{x y}^{\mu}$ have mass $1 / d)$. Thus,

$$
h(\mathfrak{M}, \mu) \leq d h(\mathfrak{M}, \widetilde{\mu}) .
$$

The measure $\widetilde{\mu}$ is a convex combination of the idempotent measures $\mu_{A}$ and $\mu_{B}$, so that its convolution powers are essentially convex combinations of the convolution powers of $\mu$. The total number of $m_{B}$ 's in the $n$-fold convolution of $\widetilde{\mu}$ is $\sim n / d$, but some of them disappear because $m_{B} m_{B}=m_{B}$, so that in fact $\widetilde{\mu}^{* n}$ is the convolution of about $\frac{d-1}{d^{2}} n$ copies of $\mu=m_{A} * m_{B}$. Thus,

$$
h(\mathfrak{M}, \widetilde{\mu})=\frac{d-1}{d^{2}} h(\mathfrak{M}, \mu),
$$

whence $h(\mathfrak{M}, \mu)=0$.

\section{REFERENCES}

[ADR00] Claire Anantharaman-Delaroche and Jean Renault, Amenable groupoids, Monographies de L'Enseignement Mathématique [Monographs of L'Enseignement Mathématique], vol. 36, L'Enseignement Mathématique, Geneva, 2000, With a foreword by Georges Skandalis and Appendix B by E. Germain. MR 2001m:22005

[Ano94] D. V. Anosov, On N. N. Bogolyubov's contribution to the theory of dynamical systems, Uspekhi Mat. Nauk 49 (1994), no. 5(299), 5-20. MR 1311227 (96a:01029)

[Ave72] André Avez, Entropie des groupes de type fini, C. R. Acad. Sci. Paris Sér. A-B 275 (1972), A1363-A1366. MR 0324741 (48 \#3090)

[BG00] L. Bartholdi and R. I. Grigorchuk, On the spectrum of Hecke type operators related to some fractal groups, Tr. Mat. Inst. Steklova 231 (2000), no. Din. Sist., Avtom. i Beskon. Gruppy, 5-45. MR 1841750 (2002d:37017)

[BG02] Laurent Bartholdi and Rostislav Grigorchuk, On a group associated to $z^{2}-1$, arXiv: math.GR/0203244, 2002.

[BGN03] Laurent Bartholdi, Rostislav Grigorchuk, and Volodymyr Nekrashevych, From fractal groups to fractal sets, Fractals in Graz 2001, Trends Math., Birkhäuser, Basel, 2003, pp. 25-118. MR 2091700 (2005h:20056)

[Bie90] Ben Bielefeld (ed.), Conformal dynamics problem list, Institute for Mathematical Sciences preprint series, no. 90-1, SUNY Stony Brook, 1990, arXiv: math.DS/9201271.

[BKN08] Laurent Bartholdi, Vadim Kaimanovich, and Volodymyr Nekrashevych, On amenability of automata groups, arXiv:0802.2837, 2008.

[BN03] E. Bondarenko and V. Nekrashevych, Post-critically finite self-similar groups, Algebra Discrete Math. (2003), no. 4, 21-32. MR 2070400 (2005d:20041) 
[BN06] Laurent Bartholdi and Volodymyr Nekrashevych, Thurston equivalence of topological polynomials, Acta Math. 197 (2006), no. 1, 1-51. MR 2285317 (2008c:37072)

[Bog39] N. N. Bogolyubov, On some ergodic properties of continuous transformation groups, Nauch. Zap. Kiev Univ. Phys.-Mat. Sb. 4 (1939), no. 5, 45-52, in Ukranian, also Selected works in mathematics, Fizmatlit, Moscow, 2006, pp. 213-222 (in Russian).

[BS98] A. M. Brunner and Said Sidki, The generation of $\mathrm{GL}(n, \mathbb{Z})$ by finite state automata, Internat. J. Algebra Comput. 8 (1998), no. 1, 127-139. MR 1492064 (99f:20055)

[BV05] Laurent Bartholdi and Bálint Virág, Amenability via random walks, Duke Math. J. 130 (2005), no. 1, 39-56. MR 2176547 (2006h:43001)

[CSGdlH99] Tullio Ceccherini-Silberstein, Rostislav I. Grigorchuk, and Pierre de la Harpe, Amenability and paradoxical decompositions for pseudogroups and discrete metric spaces, Proc. Steklov Inst. Math. 224 (1999), no. 1, 57-97. MR 1721355 (2001h:43001)

[CW89] A. Connes and E. J. Woods, Hyperfinite von Neumann algebras and Poisson boundaries of time dependent random walks, Pacific J. Math. 137 (1989), no. 2, 225-243. MR 90h:46100

[Day49] Mahlon M. Day, Means on semigroups and groups, Bull. Amer. Math. Soc. 55 (1949), 10541055, abstract 55-11-507.

[Day57] — Amenable semigroups, Illinois J. Math. 1 (1957), 509-544. MR 0092128 (19,1067c)

[Day83] C Citation classic - amenable semigroups, Current Contents Phys. Chem. Earth (1983), no. 26, 18-18.

[Der76] Yves Derriennic, Lois "zéro ou deux" pour les processus de Markov. Applications aux marches aléatoires, Ann. Inst. H. Poincaré Sect. B (N.S.) 12 (1976), no. 2, 111-129. MR 54 \#11508

[Der80] - Quelques applications du théorème ergodique sous-additif, Conference on Random Walks (Kleebach, 1979) (French), Astérisque, vol. 74, Soc. Math. France, Paris, 1980, pp. 183-201, 4. MR 588163 (82e:60013)

[dlH00] Pierre de la Harpe, Topics in geometric group theory, Chicago Lectures in Mathematics, University of Chicago Press, Chicago, IL, 2000. MR 1786869 (2001i:20081)

[Fal03] Kenneth Falconer, Fractal geometry, second ed., John Wiley \& Sons Inc., Hoboken, NJ, 2003, Mathematical foundations and applications. MR 2118797 (2006b:28001)

[Fø155] Erling Følner, On groups with full Banach mean value, Math. Scand. 3 (1955), 243-254. MR $0079220(18,51 \mathrm{f})$

[Fur73] Harry Furstenberg, Boundary theory and stochastic processes on homogeneous spaces, Harmonic analysis on homogeneous spaces (Proc. Sympos. Pure Math., Vol. XXVI, Williams Coll., Williamstown, Mass., 1972), Amer. Math. Soc., Providence, R.I., 1973, pp. 193-229. MR $50 \# 4815$

[GM05] Yair Glasner and Shahar Mozes, Automata and square complexes, Geom. Dedicata 111 (2005), 43-64. MR 2155175 (2006g:20112)

[GN07] Rostislav Grigorchuk and Volodymyr Nekrashevych, Self-similar groups, operator algebras and Schur complement, J. Mod. Dyn. 1 (2007), no. 3, 323-370. MR 2318495 (2008e:46072)

[Gre69] Frederick P. Greenleaf, Invariant means on topological groups and their applications, Van Nostrand Mathematical Studies, No. 16, Van Nostrand Reinhold Co., New York, 1969. MR $40 \# 4776$

[Gri80] R. I. Grigorchuk, On Burnside's problem on periodic groups, Funktsional. Anal. Appl. 14 (1980), no. 1, 41-43. MR 565099 (81m:20045)

[Gri85] - Degrees of growth of finitely generated groups and the theory of invariant means, Math. SSSR Izv. 25 (1985), no. 2, 259-300. MR 764305 (86h:20041)

[Gri98] - An example of a finitely presented amenable group that does not belong to the class EG, Sb. Math. 189 (1998), no. 1-2, 75-95. MR 1616436 (99b:20055)

[Gro81] Mikhael Gromov, Groups of polynomial growth and expanding maps, Inst. Hautes Études Sci. Publ. Math. (1981), no. 53, 53-73. MR 623534 (83b:53041)

[GŻ02a] Rostislav I. Grigorchuk and Andrzej Żuk, On a torsion-free weakly branch group defined by a three state automaton, Internat. J. Algebra Comput. 12 (2002), no. 1-2, 223-246, International Conference on Geometric and Combinatorial Methods in Group Theory and Semigroup Theory (Lincoln, NE, 2000). MR 2003c:20048

[GŻ02b] - Spectral properties of a torsion-free weakly branch group defined by a three state automaton, Computational and statistical group theory (Las Vegas, NV/Hoboken, NJ, 2001), Contemp. Math., vol. 298, Amer. Math. Soc., Providence, RI, 2002, pp. 57-82. MR 2003h:60011 
[Kai92] Vadim A. Kaimanovich, Measure-theoretic boundaries of Markov chains, 0-2 laws and entropy, Harmonic analysis and discrete potential theory (Frascati, 1991), Plenum, New York, 1992, pp. 145-180. MR 94h:60099

[Kai95] _ The Poisson boundary of covering Markov operators, Israel J. Math. 89 (1995), no. 1-3, 77-134. MR 96k:60194

[Kai03] _ Random walks on Sierpiński graphs: hyperbolicity and stochastic homogenization, Fractals in Graz 2001, Trends Math., Birkhäuser, Basel, 2003, pp. 145-183. MR 2091703 (2005h:28022)

[Kai05] _ "Münchhausen trick" and amenability of self-similar groups, Internat. J. Algebra Comput. 15 (2005), no. 5-6, 907-937. MR 2197814

[KB37] Nicolas Kryloff and Nicolas Bogoliouboff, La théorie générale de la mesure dans son application à l'étude des systèmes dynamiques de la mécanique non linéaire, Ann. of Math. (2) 38 (1937), no. 1, 65-113. MR 1503326

[Kig01] Jun Kigami, Analysis on fractals, Cambridge Tracts in Mathematics, vol. 143, Cambridge University Press, Cambridge, 2001. MR 1840042 (2002c:28015)

[KS83] András Krámli and Domokos Szász, Random walks with internal degrees of freedom. I. Local limit theorems, Z. Wahrsch. Verw. Gebiete 63 (1983), no. 1, 85-95. MR 85f:60098

[KV83] V. A. Kaimanovich and A. M. Vershik, Random walks on discrete groups: boundary and entropy, Ann. Probab. 11 (1983), no. 3, 457-490. MR 85d:60024

[Nek05] Volodymyr Nekrashevych, Self-similar groups, Mathematical Surveys and Monographs, vol. 117, American Mathematical Society, Providence, RI, 2005. MR 2162164 (2006e:20047)

[Nek08]_ Free subgroups in groups acting on rooted trees, arXiv:0802.2554, 2008.

[NT08] Volodymir Nekrashevych and Alexander Teplyaev, Groups and analysis on fractals, Analysis on Graphs and its Applications (Proc. Sympos. Pure Math., Vol. 77), Amer. Math. Soc., Providence, R.I., 2008, pp. 143-180.

[Pat88] Alan L. T. Paterson, Amenability, Mathematical Surveys and Monographs, vol. 29, American Mathematical Society, Providence, RI, 1988. MR 90e:43001

[Pie84] Jean-Paul Pier, Amenable locally compact groups, Pure and Applied Mathematics, John Wiley \& Sons Inc., New York, 1984, A Wiley-Interscience Publication. MR 86a:43001

[Rei65] H. Reiter, On some properties of locally compact groups, Nederl. Akad. Wetensch. Proc. Ser. A 68=Indag. Math. 27 (1965), 697-701. MR 0194908 (33 \#3114)

[Roh67] V. A. Rohlin, Lectures on the entropy theory of transformations with invariant measure, Uspehi Mat. Nauk 22 (1967), no. 5 (137), 3-56. MR 0217258 (36 \#349)

[Ros81] Joseph Rosenblatt, Ergodic and mixing random walks on locally compact groups, Math. Ann. 257 (1981), no. 1, 31-42. MR 83f:43002

[RT09] Luke G. Rogers and Alexander Teplyaev, Laplacians on the Basilica Julia set, Commun. Pure Appl. Anal. (2009), to appear.

[Sid00] Said Sidki, Automorphisms of one-rooted trees: growth, circuit structure, and acyclicity, J. Math. Sci. (New York) 100 (2000), no. 1, 1925-1943, Algebra, 12. MR 1774362 (2002g:05100)

[VK79] A.M. Vershik and V. A Kaimanovich, Random walks on groups: boundary, entropy, uniform distribution, Dokl. Akad. Nauk SSSR 249 (1979), no. 1, 15-18. MR 553972 (81f:60098)

[vN29] John von Neumann, Zur allgemeinen Theorie des Maßes, Fund. Math. 13 (1929), 73-116 and 333, also Collected works, vol. I, pages 599-643.

[VV07] Mariya Vorobets and Yaroslav Vorobets, On a free group of transformations defined by an automaton, Geom. Dedicata 124 (2007), 237-249. MR 2318547 (2008i:20030)

[YY83] A. M. Yaglom and I. M. Yaglom, Probability and information, Theory and Decision Library, vol. 35, D. Reidel Publishing Co., Dordrecht, 1983, Translated from the third Russian edition by V. K. Jain. MR 736349 (85d:94001)

Jacobs University Bremen, 28759 Bremen, Germany

E-mail address: vadim.kaimanovich@gmail.com 